\section{Estudo comparativo de dois critérios utilizados no Programa Saúde da Família na priorização do tratamento da cárie entre crianças de} 5 a 12 anos

\author{
Comparative study of two classification criteria \\ used in the Family Health Program to prioritize \\ treatment of dental caries in 5-to-12 year-old \\ children
}

André Luiz Marçal Terreri 1,2

Zaida Aurora Sperli Geraldes Soler 2

\footnotetext{
${ }^{1}$ Centro Universitário de Rio Preto, São José do Rio Preto, Brasil.

2 Faculdade de Medicina de São José do Rio Preto, São José do Rio Preto, Brasil.

Correspondência

A. L. M. Terreri

Centro Universitário de Rio Preto.

Rua José Nogueira de

Carvalho 410, apto. 103 São José do Rio Preto, SP 15060-100, Brasil. andreterreri@ig.com.br
}

\begin{abstract}
The objective of this study was to evaluate two classification criteria used to prioritize treatment of dental caries under the Brazilian Family Health Program. The study was held in Ipiguá, São Paulo State, and included 538 five-totwelve-year-old children. The children underwent an oral examination and assessment of two classification criteria in order to prioritize restorative dental treatment. Based on caries activity, 236 children (75.4\%) were prioritized for dental care, while the criterion based on amount and size of the cavity included 73 children (23.4\%). Using the two criteria, 186 children (59.4\%) were not classified with the same priority for restorative treatment. Determining the precedence for restorative dental treatment must be based on the caries activity, number of cavities, need for treatment, and type of dentition involved (deciduous or permanent). Subjective measures can also be used as a supplement for more rational planning.
\end{abstract}

Dental Care; Dental Caries; Family Health Program; Single Health System
Introdução

As diretrizes da política nacional de saúde bucal orientam para que as ações e serviços odontológicos resultem de um adequado conhecimento da realidade de saúde de cada localidade para, valendo-se disso, construir uma prática efetivamente resolutiva 1 .

Tem sido discutido que a odontologia instituída no âmbito do Programa Saúde da Família (PSF) constitui-se em uma importante ferramenta para organizar a oferta dos serviços odontológicos curativos e preventivos, considerando-se as informações obtidas no cadastramento das condições de saúde bucal realizado durante as visitas domiciliares. Entretanto, algumas equipes de saúde bucal demonstram dificuldades de adaptação à nova lógica de organização da oferta dos serviços odontológicos. Lourenço 2 pesquisou no Estado de Minas Gerais e verificou que $74,1 \%$ das equipes de saúde bucal utilizavam mais de uma forma de agendamento dos usuários, e 39,9\% admitiam a livre oferta dos serviços odontológicos como porta de entrada para o atendimento no PSF e apenas $26,9 \%$ utilizavam a triagem de risco como forma de priorização à prestação desses serviços. No Estado do Paraná, Baldani et al. ${ }^{3}$ observaram que a livre oferta é mantida em 9,5\% das equipes de saúde bucal, como opção de prestação dos serviços odontológicos. Em municípios do Estado da Paraíba, Padilha et al. 4 também encontraram equipes de saúde bucal que prestavam 
atendimento eminentemente curativo conforme a procura espontânea dos usuários.

No Brasil, a conseqüência da prestação dos serviços por meio da livre demanda somada aos problemas de exclusão social, econômicos e educacionais é o estabelecimento de um padrão cultural de procura por serviços odontológicos quando a doença já se estabeleceu e requer tratamento curativo e alívio da dor. Tal realidade pôde ser constatada durante a realização do levantamento das condições de saúde bucal da população brasileira 2002-2003, sendo relatada a dor dentária para procura do dentista por $30 \%$ de adolescentes e $46 \%$ de adultos e idosos 5 . Para que ocorra uma mudança nesse padrão cultural de procura dos serviços por causa da presença de dor, é necessário que haja, também, uma mudança real no modelo de organização da demanda. A equipe de saúde bucal inserida na estratégia do PSF deve realizar o cadastramento em saúde bucal no domicílio e fazer uso das informações obtidas no desenvolvimento de atividades preventivas, educativas e curativas. No que diz respeito à cárie dentária, em algumas localidades do Estado de São Paulo e do Distrito Federal foram definidos critérios para serem utilizados no contexto do PSF, para organizar a priorização dos tratamentos curativos. O critério de classificação sugerido pela Secretaria de Estado de Saúde de São Paulo 6 considera a atividade doença cárie para determinar a prioridade no atendimento, enquanto o critério usado pela Secretaria de Estado de Saúde do Distrito Federal 7 tem como referência a quantidade e o tamanho da cavidade de cárie dentária.

Frente às diversidades na maneira de priorização para o tratamento da cárie dentária, o objetivo deste estudo foi avaliar os dois critérios utilizados no cadastramento das condições de saúde bucal, da Secretaria de Estado de Saúde de São Paulo e da Secretaria de Estado de Saúde do Distrito Federal, apontando suas tendências na forma de priorizar o atendimento odontológico curativo para crianças.

\section{Métodos}

O campo de estudo para a realização desta pesquisa foi o Município de Ipiguá, São Paulo, Brasil, com população estimada de 4.195 habitantes em 2005, segundo o Instituto Brasileiro de Geografia e Estatística (IBGE) 8. Foram estudadas crianças na faixa etária entre 5 a 12 anos de idade, e que de acordo com as estimativas estratificadas por idade do IBGE, havia 602 crianças residentes no município. Dessas, 583 crianças estavam matriculadas na única escola do município. O grupo estu- dado correspondeu a 538 (89,3\%) crianças, sendo a totalidade daqueles que não faltaram à escola nos dias dos exames definidos neste estudo e que trouxeram devidamente preenchido e assinado pelos pais ou responsáveis o termo de consentimento (cujo modelo foi previamente aprovado pelo Comitê de Ética em Pesquisa do Instituto do Coração de Rio Preto, preenchendo os requisitos fundamentais da Resolução no. 196/96 do Conselho Nacional de Ética em Pesquisa), distribuído previamente pelos professores com apoio das Secretarias Municipais de Educação e de Saúde. Nove crianças não participaram da amostragem em razão da ausência nos dias dos exames, e outras 36 por não apresentarem o consentimento dos responsáveis para a sua realização.

Como procedimento de coleta dos dados, foi realizado o exame bucal nas crianças incluídas no estudo e preenchimento dos dois critérios de cadastramento das condições de saúde bucal usados para se determinar a prioridade do tratamento restaurador da cárie. Como o objeto desta pesquisa foi a organização da demanda para o atendimento clínico curativo da cárie, foram consideradas apenas as informações relativas à presença de lesão cavitada, descartando as outras informações contidas nas duas fichas de cadastramento familiar.

Vale lembrar que os dois critérios aqui estudados são utilizados no âmbito do PSF, sendo um usado pela Secretaria de Estado de Saúde de São Paulo e o outro, pela Secretaria de Estado de Saúde do Distrito Federal.

O critério proposto pela Secretaria de Estado de Saúde de São Paulo apresenta seis códigos para classificar a situação encontrada durante o exame de triagem: código A (ausência de lesão); código B (história de dente restaurado); código C (cavidade de cárie crônica); código D (presença de placa, gengivite e mancha branca ativa); código $\mathrm{E}$ (cavidade de cárie aguda) e código $\mathrm{F}$ (presença de dor).

Nesse modelo de cadastramento, a determinação do atendimento clínico individual para a cárie, na unidade de saúde da família, teria como prioridade os indivíduos classificados como código F, seguidos, conforme a possibilidade, pelos classificados como código E (alto risco; cavidade de cárie com cor amarelada, aspecto úmido e amolecido) e, finalmente, os classificados como código C (risco moderado, cavidade de cárie com cor escurecida, brilhante, aspecto endurecido, considerando-se também os dentes permanentes com restaurações provisórias). A presença de cavidade aguda e crônica na mesma criança foi classificada a pior situação de risco (cárie aguda). Todas as crianças que não apresentaram cavidades de cárie (códigos A, B, e D), ou seja, sem 
necessidade de tratamento curativo foram excluídas, uma vez que o objeto de estudo da pesquisa foi a priorização do tratamento curativo.

O modelo de ficha proposto pela Secretaria de Estado de Saúde do Distrito Federal tem como critério para a prioridade de tratamento curativo a presença de dor, a quantidade e o tamanho da cavidade de cárie dentária que necessita de tratamento restaurador, segundo a situação individual. O critério possui três códigos para classificar a situação encontrada durante o exame de triagem: código 1 (sem necessidade de tratamento); código 2 (o número de cavidades nesse código é de uma a cinco); e código 3 (mais de cinco cavidades, e/ou dor e/ou uma cavidade grande).

Os indivíduos de código 3 têm prioridade de tratamento sobre os indivíduos classificados com o código 2. Como nesse modelo não há um critério para definir "cavidade grande", convencionou-se neste estudo classificá-la como todo dente que necessitasse de tratamento pulpar ou extração. Da mesma forma que o critério anterior, as crianças que não apresentavam cavidades de cárie com necessidade de tratamento odontológico curativo (código 1) foram eliminadas das comparações entre os critérios de priorização do tratamento curativo.

Antes da coleta dos dados foram pré-selecionadas vinte crianças, de tal forma que apresentassem, coletivamente, todas as condições esperadas da população a ser examinada, para a realização de exames em duplicatas. Após o período de uma semana, as mesmas crianças foram reexaminadas para aferir os erros intraexaminador, mediante cálculo da taxa de concordância e estatística kappa (k) para os critérios de priorização do atendimento. Nesta amostra, a concordância intra-examinador e a estatística kappa alcançadas para os critérios foram, respectivamente, $93 \%$ e 0,90 .

O exame foi realizado sempre na mesma área das escolas, sob luz natural, com auxílio de espelho plano e sonda exploradora, ficando o examinador sentado e a criança, a sua frente, examinada em pé. Todas as crianças que participaram deste estudo receberam uma escova dentária e dentifrício, doados por uma empresa de produtos de higiene pessoal, o que permitiu que fosse realizada a escovação prévia ao exame.

O cirurgião dentista examinador foi o próprio autor deste estudo, que também orientou e supervisionou o registro das informações, feito por uma auxiliar de consultório dentário. Com o intuito de detectar a ocorrência de mudanças na forma de aplicar os critérios, a cada 100 crianças examinadas, dez exames em duplicata foram realizados e aplicado o cálculo da taxa de concordância e a estatística kappa. Os dados ob- tidos foram agrupados segundo características de semelhanças, tratados em função de índices absolutos e percentuais, e são apresentados em forma de tabelas.

\section{Resultados}

Como mencionado, foram realizados 538 exames bucais em escolares de 5 e 12 anos do Município de Ipiguá. Para medir a concordância intraexaminador no decorrer dos exames realizados, foram reexaminados cinqüenta escolares, obtendo-se $94 \%$ de concordância global entre os exames e reexames, com índice da kappa de 0,92.

A Tabela 1 mostra a distribuição das 538 crianças, na faixa etária de 5 a 12 anos de idade, quanto à necessidade de tratamento curativo de cárie, observando-se que $313(58,1 \%)$ apresentavam alguma necessidade de intervenção restauradora curativa.

O preenchimento dos dois modelos de fichas para as mesmas crianças permitiu verificar, conforme os dados da Tabela 2, que no grupo das crianças classificadas como primeira prioridade para o tratamento, o critério adotado pela Secretaria de Estado de Saúde de São Paulo, que se baseia na atividade da doença, determinou $75,4 \%$ das crianças como primeira prioridade, enquanto o critério da Secretaria de Estado de Saúde do Distrito Federal determinou 76,6\% das crianças como segunda prioridade para atendimento.

A Tabela 3 revela a distribuição de 313 crianças, presentes ou não na mesma prioridade, de acordo com os dois critérios de priorização do atendimento. Verifica-se que a maioria das crian-

Tabela 1

Prevalência de escolares na faixa etária 5 a 12 anos, segundo necessidade de tratamento curativo para cárie. Ipiguá, São Paulo, Brasil, 2005.

\begin{tabular}{lccc}
\hline Idade (anos) & $\begin{array}{c}\text { Quantidade de crianças } \\
\text { examinadas }\end{array}$ & $\begin{array}{c}\text { Quantidade de crianças com necessidade } \\
\text { de tratamento curativo para cárie }\end{array}$ \\
& & $\mathbf{n}$ & \\
\hline 5 & 53 & 27 & 50,9 \\
6 & 78 & 55 & 70,5 \\
7 & 64 & 47 & 73,4 \\
8 & 53 & 36 & 67,9 \\
9 & 82 & 50 & 60,9 \\
10 & 68 & 40 & 58,8 \\
11 & 62 & 27 & 43,5 \\
12 & 78 & 31 & 39,7 \\
Total & 538 & 313 & 58,1 \\
\hline
\end{tabular}


Tabela 2

Número e percentual de crianças de 5 a 12 anos quanto à prioridade no tratamento curativo para cárie, segundo os critérios das Secretarias de Estado de Saúde de São Paulo e do Distrito Federal.

\begin{tabular}{|c|c|c|c|c|c|c|c|c|c|}
\hline \multirow[t]{3}{*}{$\begin{array}{l}\text { Idade } \\
\text { (anos) }\end{array}$} & \multirow{3}{*}{$\begin{array}{l}\text { Quantidade de crianças } \\
\text { com necessidade } \\
\text { de tratamento }\end{array}$} & \multicolumn{4}{|c|}{$\begin{array}{l}\text { Critério da Secretaria de } \\
\text { Estado de Saúde de São Paulo }\end{array}$} & \multicolumn{4}{|c|}{$\begin{array}{l}\text { Critério da Secretaria de } \\
\text { Estado de Saúde do Distrito Federal }\end{array}$} \\
\hline & & \multicolumn{2}{|c|}{ Primeira prioridade } & \multicolumn{2}{|c|}{ Segunda prioridade } & \multicolumn{2}{|c|}{ Primeira prioridade } & \multicolumn{2}{|c|}{ Segunda prioridade } \\
\hline & & $\mathrm{n}$ & $\%$ & $n$ & $\%$ & $\mathrm{n}$ & $\%$ & $\mathbf{n}$ & $\%$ \\
\hline 5 & 27 & 19 & 70,3 & 8 & 29,7 & 9 & 33,3 & 18 & 66,6 \\
\hline 6 & 55 & 49 & 89,1 & 6 & 10,9 & 22 & 40,0 & 33 & 60,0 \\
\hline 7 & 47 & 42 & 89,3 & 5 & 10,7 & 16 & 34,0 & 31 & 66,0 \\
\hline 8 & 36 & 28 & 77,8 & 8 & 22,2 & 10 & 27,7 & 26 & 72,3 \\
\hline 9 & 50 & 35 & 70,0 & 15 & 30,0 & 8 & 16,0 & 42 & 84,0 \\
\hline 10 & 40 & 27 & 67,5 & 13 & 32,5 & 4 & 10,0 & 36 & 90,0 \\
\hline 11 & 27 & 16 & 59,2 & 11 & 40,8 & 2 & 7,4 & 25 & 92,6 \\
\hline 12 & 31 & 20 & 64,5 & 11 & 35,5 & 2 & 6,4 & 29 & 93,6 \\
\hline 5-12 & 313 & 236 & 75,4 & 77 & 24,6 & 73 & 23,4 & 240 & 76,6 \\
\hline
\end{tabular}

Tabela 3

Número e percentual de crianças de 5 a 12 anos, presentes ou não na mesma prioridade, segundo os critérios de tratamento curativo de cárie das Secretarias de Estado de Saúde de São Paulo e do Distrito Federal.

\begin{tabular}{|c|c|c|c|c|c|c|c|}
\hline \multirow[t]{3}{*}{$\begin{array}{l}\text { Idade } \\
\text { (anos) }\end{array}$} & \multirow{3}{*}{$\begin{array}{l}\text { Quantidade de crianças } \\
\text { com necessidade } \\
\text { de tratamento }\end{array}$} & \multicolumn{4}{|c|}{$\begin{array}{c}\text { Crianças presentes na mesma } \\
\text { prioridade }\end{array}$} & \multicolumn{2}{|c|}{$\begin{array}{c}\text { Crianças não presentes na } \\
\text { mesma prioridade }\end{array}$} \\
\hline & & \multicolumn{2}{|c|}{ Primeira prioridade } & \multicolumn{2}{|c|}{ Segunda prioridade } & \multirow[b]{2}{*}{$\mathrm{n}$} & \multirow[b]{2}{*}{$\%$} \\
\hline & & $\mathbf{n}$ & $\%$ & $n$ & $\%$ & & \\
\hline 5 & 27 & 5 & 18,5 & 2 & 7,4 & 20 & 74,0 \\
\hline 6 & 55 & 20 & 36,3 & 4 & 7,2 & 31 & 56,3 \\
\hline 7 & 47 & 14 & 29,7 & 3 & 6,3 & 30 & 63,8 \\
\hline 8 & 36 & 7 & 19,4 & 6 & 16,6 & 23 & 63,8 \\
\hline 9 & 50 & 8 & 16,0 & 15 & 30,0 & 27 & 54,0 \\
\hline 10 & 40 & 4 & 10,0 & 13 & 32,5 & 23 & 57,5 \\
\hline 11 & 27 & 1 & 3,7 & 10 & 37,0 & 16 & 59,2 \\
\hline 12 & 31 & 2 & 6,4 & 11 & 35,4 & 18 & 58,0 \\
\hline Total & 313 & 61 & 19,4 & 66 & 21,0 & 186 & 59,4 \\
\hline
\end{tabular}

ças $(59,4 \%)$ não coincidiu na determinação da prioridade do tratamento curativo: 61 crianças $(19,4 \%)$ coincidiram na primeira prioridade e 66 crianças $(21 \%)$ foram coincidentes na segunda prioridade. A análise dos dados permite afirmar que a concordância dos critérios para determinar a prioridade de tratamento é baixa.

\section{Discussão}

Apesar de os estudos demonstrarem que a prevalência da doença cárie melhorou nas últimas dé- cadas no Brasil, tem-se verificado que a melhoria vem sendo acompanhada pela polarização do agravo 9,10, sendo relatada com freqüência na literatura, em grupos de população submetidos à privação social 11,12,13,14. O fato de a doença cárie concentrar índices mais altos em pequenos grupos populacionais mais desfavorecidos do ponto de vista social, econômico e educacional, dentro de um mesmo país, região, município e até mesmo dentro da área de abrangência da equipe de saúde da família, indica a necessidade de iniciar o cadastramento das condições de saúde bucal nas microáreas de maior risco. Dessa forma, em 
locais em que já existe equipe de saúde da família em atividade, e nos quais a equipe de saúde bucal está sendo implantada, seria útil a análise das informações contidas na "Ficha A" do sistema de informação da atenção básica. A ficha contém informações - das famílias e seus indivíduos sobre saúde (morbidade referida), escolaridade, ocupação, tipo de casa, forma de abastecimento de água, destino do lixo, entre outras informações relevantes na identificação das microáreas de risco e das famílias a elas pertencentes. As famílias de alto risco deverão ser as primeiras a participar do cadastramento das condições de saúde bucal, para a identificação do risco individual e, em seguida, inseridas no grupo da assistência odontológica curativa 15. A análise das informações do risco familiar ("Ficha A") e do risco individual, obtida durante o cadastramento, também é importante no planejamento das ações educativas e preventivas que devem ser desenvolvidas no âmbito domiciliar.

Neste estudo, como se verifica na Tabela 2, quando o critério proposto pela Secretaria de Estado de Saúde de São Paulo foi aplicado para determinar a priorização do tratamento clínico das crianças com lesões cavitadas, a situação aguda foi a mais encontrada. Assim, constatouse um grupo grande de crianças como primeira prioridade para receber o tratamento odontológico, fato que, possivelmente, ocorreria nas microáreas onde há famílias de maior risco. A esse critério seria útil acrescentar outros aspectos, no momento de determinar uma ordem de encaminhamento das crianças, como o número de dentes com cavidades, o tratamento necessário e o tipo de dente acometido (permanente ou decíduo). Com relação à quantidade de dentes com lesão cavitada, é evidente que, quanto maior o número de lesões cavitadas em situação aguda que uma criança apresentar, maior a prioridade para receber o tratamento odontológico. O número de cavidades é um aspecto abordado no critério usado pela Secretaria de Estado de Saúde do Distrito Federal, que quantifica, por meio de seus códigos, as cavidades de cárie para cada criança examinada. Entretanto, esse critério não estabelece distinção entre as crianças com as seqüelas da doença e as crianças com atividade da doença, o que pode ocasionar situações em que lesões cavitadas agudas se agravem no decorrer da espera para o tratamento, aumentando a complexidade e o custo do procedimento. Por outro lado, durante a realização do exame, constataram-se crianças com extensas destruições coronárias, ocasionadas provavelmente por cárie de mamadeira nos primeiros anos de vida, mas que estavam inativas (lesões crônicas) no momento. Conforme o critério adotado pela Se- cretaria de Estado de Saúde de São Paulo, crianças que possuírem uma lesão cavitada aguda terão prioridade no atendimento odontológico sobre aquelas que possuírem lesões cavitadas em situação crônica, independente do número de cavidades. É importante ressaltar que crianças portadoras de cárie severa têm problemas para comer certos alimentos, faltam às aulas por causa dos dentes e tem seu desempenho escolar comprometido 16 .

Neste caso, pode-se optar por estipular cotas de atendimento odontológico na unidade de saúde da família, tendo como parâmetro a proporção de crianças classificadas nos grupos lesão cavitada de cárie aguda (código E) e lesão cavitada de cárie crônica (código C).

Como critério de desempate entre crianças com o mesmo número de cavidades seria importante identificar do tipo de tratamento (restaurador, pulpar e extração) mais adequado, em conformidade com os procedimentos realizados na unidade de saúde da família. Nesse caso, o tratamento restaurador teria um peso maior na determinação da prioridade, seguido das necessidades de tratamento pulpar mais restaurador, seguido por sua vez, das necessidades de extração. Com relação às necessidades de tratamento é necessário fazer a distinção entre a dentição permanente e a decídua, aspecto que auxiliaria na determinação do atendimento, já que a dentição permanente deve ser priorizada, especialmente quando temos uma grande população adscrita. É importante salientar que o tratamento restaurador, por si só, não garante o controle do desenvolvimento da doença cárie no indivíduo, bem como não ocasiona redução da prevalência de cárie na população. A doença só pode ser estacionada com medidas que interfiram na progressão da doença tais como; a remoção regular da placa bacteriana, controle da dieta e terapia tópica de fluoreto, ou seja, em medidas não operatórias 17.

Os autores também sugerem que estudos futuros devam contemplar, na organização da oferta dos serviços, além dos métodos tradicionais de exame clínico (critérios normativos), o uso de dimensões não normativas (impactos da saúde bucal sobre a qualidade de vida e atividades diárias). Isso porque, em decorrência de problemas odontológicos, crianças podem ter suas atividades diárias prejudicadas, em razão de: diminuição das horas de sono, não-realização de algumas atividades de lazer, restrições alimentares, desordens psicológicas relacionadas à queda da auto-estima e perda de dias e de aprendizagem na escola 18 .

O uso somente de critérios normativos apresenta limitações consideráveis. Um dos grandes 
problemas dessa abordagem normativa é que ela não considera o modo como as pessoas realmente se sentem e, portanto, não se aproxima dos conceitos mais atuais de saúde. Os critérios não normativos devem ser usados de forma complementar, e não em substituição ao exame clínico, com a finalidade de aproximar-se mais das necessidades de saúde e focar mais na melhoria da qualidade de vida das populações 19 .

Verificou-se nesta pesquisa, como mostrado na Tabela 3, uma baixa concordância entre os dois critérios utilizados, fato que revela a necessidade de mais estudos neste sentido, como forma de aproveitar as experiências odontológicas no âmbito do PSF que vêm sendo adotadas, objetivando a padronização de um modelo de cadastro nacional em saúde bucal.

Sugere-se a elaboração de um critério que deva abordar a atividade da doença, o número de cavidades com distinção de dentes permanentes e decíduos, as necessidades de tratamento, além da inclusão de uma abordagem não normativa que auxilie identificar os impactos das condições de saúde bucal sobre a qualidade de vida e atividades diárias.

\section{Resumo}

O objetivo deste trabalho foi avaliar dois critérios utilizados no Programa Saúde da Família para determinação da prioridade do atendimento odontológico curativo da cárie. O estudo foi feito no Município de Ipiguá, São Paulo, Brasil, entre 538 crianças de 5 a 12 anos de idade. Realizou-se o exame bucal e preenchimento de dois critérios de classificação para as mesmas crianças a fim de avaliar as prioridades no atendimento odontológico curativo resultantes de cada um dos critérios. Com base na atividade da doença cárie, verificou-se que 236 crianças $(75,4 \%)$ foram consideradas como primeira prioridade no atendimento odontológico, enquanto o critério baseado na quantidade e tamanho da cavidade de cárie incluiu 73 crianças (23,4\%). Conforme os critérios, 186 crianças $(59,4 \%)$ não estiveram presentes na mesma prioridade de tratamento curativo para a cárie. Para determinar a prioridade do atendimento odontológico da cárie é necessário considerar além da atividade da doença e da quantidade de cavidades, a necessidade de tratamento e o tipo de dente envolvido (decíduo ou permanente). Critérios não normativos também poderiam ser usados de forma complementar para um planejamento mais racional.

Assistência Odontológica; Cárie Dentária; Programa Saúde da Família; Sistema Único de Saúde

\section{Conclusões}

Os critérios definidos pelas Secretarias de Saúde de São Paulo e do Distrito Federal, utilizados nesta pesquisa no enfoque do PSF, mostraramse muito diferentes na determinação da prioridade do atendimento odontológico curativo da cárie.

A priorização do atendimento odontológico deve analisar de forma conjunta o risco familiar e o risco individual da criança. A análise do risco familiar pode ser obtida por meio da "Ficha A" e das informações provenientes dos agentes comunitários de saúde. Para organizar a oferta dos serviços curativos, deve considerar a atividade da doença, quantificar as cavidades na dentição decídua e permanente, estabelecer os tipos de tratamento. As dimensões não normativas podem complementar os achados do exame clínico. Outras pesquisas neste contexto devem permitir a padronização do cadastramento das condições de saúde bucal de crianças usuárias do PSF.

\section{Colaboradores}

A. L. M. Terreri participou da coleta, análise de dados e redação do manuscrito. Z. A. S. G. Soler participou da orientação, análise de dados e redação do manuscrito. 


\section{Referências}

1. Coordenação Nacional de Saúde Bucal, Ministério da Saúde. Diretrizes da política nacional da saúde bucal. Brasília: Coordenação Nacional de Saúde Bucal, Ministério da Saúde; 2004.

2. Lourenço EC. A inserção de equipes de saúde bucal no Programa Saúde da Família no Estado de Minas Gerais [Dissertação Mestrado]. Piracicaba: Faculdade de Odontologia de Piracicaba, Universidade Estadual de Campinas; 2005.

3. Baldani MH, Fadel CB, Possamai T, Queiroz MGS A inclusão da odontologia no Programa Saúde da Família no Estado do Paraná, Brasil. Cad Saúde Pública 2005; 21:1026-35.

4. Padilha WWN, Valença AMG, Cavalcanti AL, Gomes LB, Almeida RVD, Taveira GS. Planejamento e programação odontológicos no Programa Saúde da Família do Estado da Paraíba: estudo qualitativo. Pesqui Bras Odontopediatria Clín Integr 2005; 5:65-74.

5. Coordenação Nacional de Saúde Bucal, Ministério da Saúde. Projeto SB Brasil 2003. Condições de saúde bucal da população brasileira 2002-2003: Principais resultados. Brasília: Coordenação Nacional de Saúde Bucal, Ministério da Saúde; 2004.

6. Secretaria de Estado de Saúde de São Paulo. A organização das ações de saúde bucal na atenção básica; uma proposta para o SUS São Paulo. São Paulo: Secretaria de Estado de Saúde do Estado de São Paulo; 2001.

7. Subsecretaria de Atenção a Saúde, Secretaria de Estado de Saúde do Distrito Federal. Programa Saúde da Família: cadastro da família. Ficha A. Brasília: Secretaria de Estado de Saúde do Distrito Federal; 1999.

8. Departamento de Informática do SUS. Informações em saúde. http://tabnet.datasus.gov.br/cgi/ tabcgi.exe?ibge/cnv/poptSP.def (acessado em 02/ Jan/2005)

9. Narvai PC, Castellanos RA, Frazão P. Prevalência de cárie em dentes permanentes de escolares do município de São Paulo, SP, 1970-1996. Rev Saúde Pública 2000; 34:196-200.
10. Cardoso L, Rösing C, Kramer P, Costa CC, Costa Filho LC. Polarização da cárie em município sem água fluoretada. Cad Saúde Pública 2003; 19 237-43.

11. Baldani MH, Narvai PC, Antunes JLF. Cárie dentária e condições sócio-econômicas no Estado do Paraná, Brasil, 1996. Cad Saúde Pública 2002; 18:755-63.

12. Peres KGA, Bastos JRM, Latorre MRDO. Severidade de cárie em crianças e relação com aspectos sociais e comportamentais. Rev Saúde Pública 2000; 34:402-8.

13. Gonçalves ER, Peres MA, Marcenes W. Cárie dentária e condições sócio-econômicas: um estudo transversal com jovens de 18 anos de Florianópolis, Santa Catarina, Brasil. Cad Saúde Pública 2002; 18:699-706.

14. Baldani MH, Vasconcelos AGG, Antunes JLF. Associação do índice CPO-D com indicadores sócioeconômicos e de provisão de serviços odontológicos no Estado do Paraná, Brasil. Cad Saúde Pública 2004; 20:143-52.

15. Área Técnica de Saúde Bucal, Secretaria Municipal da Saúde de São Paulo. Diretrizes para a atenção em saúde bucal. São Paulo: Área Técnica de Saúde Bucal, Secretaria Municipal da Saúde de São Paulo; 2006.

16. Feitosa S, Colares V, Pinkham J. The psychosocial effects of severe caries in 4-year-old children in Recife, Pernambuco, Brazil. Cad Saúde Pública 2005; 21:1550-6.

17. Carvalho JC, Maltz M. Tratamento da doença carie. In: Associação Brasileira de Odontologia de Promoção de Saúde, organizador. Promoção de saúde bucal. São Paulo: Editora Artes Médicas; 2003. p. 89-105.

18. Naidoo S, Chikte UM, Sheiham A. Prevalence and impact of dental pain in 8-10-year-olds in the Western Cape. SADJ 2001; 56:521-3.

19. Gherunpong S, Sheiham A, Tsakos G. A sociodental approach to assessing children's oral health needs: integrating an oral health-related quality of life (OHRQoL) measure into oral health service planning. Bull World Health Organ 2006; 84:36-42.

Recebido em 16/Jan/2007

Versão final reapresentada em 29/Out/2007 Aprovado em 13/Nov/2007 